\title{
Defects with deep donor and acceptor levels in nanocrystals of CdTe and CdSe
}

\author{
V.N. Babentsov \\ V. Lashkaryov Institute of Semiconductor Physics, NAS of Ukraine, 45, prospect Nauky, 03028 Kyiv, Ukraine
}

\begin{abstract}
The defects in CdTe and CdSe nanocrystals were studied by comparing the photoluminescence spectra and cyclic voltammetry dependences, which enabled us to identify two main electron levels in CdTe and four in CdSe NCs. In CdTe nanocrystals these levels are: a hole trap at the energy $E_{V}+0.5 \mathrm{eV}$ and an electron trap at $E_{c}-0.5 \mathrm{eV}$. In CdSe nanocrystals, detected were two hole traps at $E_{v}+0.52 \mathrm{eV}$ and $E_{v}+0.8 \mathrm{eV}$, and two electron traps at $E_{c}-0.25 \mathrm{eV}$ and $E_{c}-0.65 \mathrm{eV}$. The $2+/ 1+$ level of $\mathrm{V}_{\mathrm{Cd}}$ or $\mathrm{Te}_{i}$ is suggested to be an acceptor, and the $2-/ 1-$ level of an antisite defect is suggested to be a donor level.
\end{abstract}

Keywords: donor, acceptor, deep levels, nanocrystal.

Manuscript received 06.06.06; accepted for publication 23.10.06.

\section{Introduction}

Nanocrystalls (NCs) of II-VI semiconductor compounds, such as CdTe, CdSe and $\mathrm{CdS}$, represent an important class of optoelectronic materials with direct bandages that vary from 1.5 to $5.5 \mathrm{eV}$. In the nanocrystalline (nc) form they demonstrate new optical properties due to their size dependent bandgap increase and splitting of the electronic levels $[1,2]$. CdTe has a small bandgap $(1.5 \mathrm{eV})$ and the largest Bohr exciton radius $(7.3 \mathrm{~nm})$, so it is expected to show a stronger quantum confinement effect and enhanced nonlinear optical properties. For example, at the similar number of photons absorbed per one $\mathrm{NC}$ of $\mathrm{CdS}$ and $\mathrm{CdTe}$ under the same excitation intensity, the nonlinear pump-emission regime easier was reached in CdTe [3].

Numerous publications describe synthesis of CdTe, $\mathrm{CdSe}$ and CdS NCs by colloidal, sol-gel, vapor routes, etc. Colloidal synthesis gives spherical particles with narrow size distribution. Among them, CdSe NCs are the most simple for synthesis, and NCs of CdS is difficult to produce. NCs often are used as dispersed in colloidal solution, in the form of solid films, or composed into various heterostructures [4-5]. Several methods are able to produce NCs on a substrate of choice: MBE, physical vapor deposition, etc. [6]. For applications, high quantum yield (QY) of the excitonic emission is very significant, and indeed, it greatly increases in NCs due to spatial confinement of excitons. Nevertheless, two factors, which are minor ones in the bulk form of these materials, can reduce the probability of radiative recombination in NCs. At low pump intensity, recombination in surface defects is the first factor, because, in the $R \approx 1.5 \mathrm{~nm} \mathrm{NC}$, around of $1 / 3$ atoms are located on a surface, and the excitonic emission dominates only in well chemically passivated NCs. Due to a small volume of a $\mathrm{NC}$, it is considered that the classical defects of lattice that exist in the bulk form, do not exist in NCs. At high pumping, non-emitting Auger recombination processes in NCs develop easier than in the bulk form of materials due to the splitted lowest Cband level; this increases probability to have more than one electron on this level.

The nature of defects that are responsible for the sub-bandgap emissions in NCs is still not fully known, also unknown is the origin of nonradiative recombination in $\mathrm{NCs}$, despite of a great afford to avoid radiative or nonradiative recombination in NCs by a proper passivation.

In our previous works, we investigated defects in colloidal NCs of CdSe by means of low temperature photoluminescence (PL), and the intensity-excitation dependencies of the excitonic and deep-level emissions in CdSe NCs at various temperatures, i.e., the behaviour of PL emission under increased pump [7,8]. Two reasons for such phenomenon in $\mathrm{CdSe} \mathrm{NCs}$ were highlighted, 1) trapping of electrons/holes on defect levels in/outside of NCs, and 2) generation of two or more electron-hole pairs per a NC. The last situation is favorable for development in NCs of non-emitting 
Auger recombination processes. Following to this line, the present work is devoted to study of donor/acceptor nature of the defect states' in the bandgap of CdTe and $\mathrm{CdSe} \mathrm{NCs}$ by comparing the low temperature photoluminescence and cyclic voltammetry spectra.

\section{Experimental}

2.1. CdTe and CdSe NCs have been synthesized according to the procedure described elsewhere [7-9]. Here we repeat it briefly. For the preparation of nc-CdSe a $100 \mathrm{ml}$ Schlenk flask containing $271.74 \mathrm{mg}$ cadmium stearate, and $4 \mathrm{~g}$ tri-n-octylphosphine oxide (TOPO), was heated under inert gas atmosphere (N2) to $250{ }^{\circ} \mathrm{C}$ and degassed several times. A solution of $23.7 \mathrm{mg}$ selenium, in $2 \mathrm{ml}$ tri-n-octylphosphine (Se/TOP) was prepared in a drybox and transferred into a syringe. The $\mathrm{Se} / \mathrm{TOP}$ solution was quickly injected into the cadmium solution under Schlenk conditions and the mixture was stirred vigorously for $3 \mathrm{~min}$ at $220^{\circ} \mathrm{C}$. The reaction mixture allowed to cool down to room temperature and the nanocrystals were precipitated with $10 \mathrm{ml}$ of dry methanol. After centrifugation, the nanoparticles were washed with dry methanol once and redissolved in dry chloroform.

The nc-CdTe has been prepared by the method described in [9]. Dissolved $\mathrm{Cd}\left(\mathrm{ClO}_{4}\right)_{2} \cdot 6 \mathrm{H}_{2} \mathrm{O}$ in water and TGA (or MPA) was adjusted the $\mathrm{pH}$ to $11.2-11.8$ by dropwise addition of $\mathrm{NaOH}$ solution. $\mathrm{H}_{2}$ Te was careered with nitrogen and passed through the solution, forming the CdTe precursor. It was converted to the nc-CdTe by refluxing the reaction mixture at $100{ }^{\circ} \mathrm{C}$. CdTe NCs of different sizes were taken from the crude solution at different refluxing times.

\subsection{Characterization methods}

The size and shape of as-prepared CdTe and CdSe NCs were determined using the transmission electron microscope (TEM), Model Zeiss LEO 912 Omega, which operated at $120 \mathrm{kV}$. The samples for the TEM study were prepared by drop casting of colloidal solutions of NCs onto carbon films, supported by a $\mathrm{Cu}$ grid. The size of NCs was inferred from the TEM images, and it was confirmed by optical absorption and PL spectroscopy.

The PL was excited at $488 \mathrm{~nm}$ with an Ar laser in the power range $10-100 \mathrm{~mW}$. The laser beam was focused onto a spot of approximately $50 \mu \mathrm{m}$ in diameter with a photo-objective which was also used for collection of the PL emission.

The samples for PL measurements were prepared by the room temperature (RT) drop casting of diluted chloroform dispersions of the CdTe and CdSe NCs onto clean glass plates in air. They were immediately placed in the dark in a pumped chamber of the cryostat with regulated temperature.

Electrochemical cyclic voltammetry measurements were performed in a three-electrode two-compartment cell with a platinum counter electrode and an $\mathrm{Ag} / \mathrm{AgCl} / \mathrm{KCl}$ (sat.) electrode as the reference one. The reference electrode potential is used to determine the relative potentials, which were controlled by a conventional potentiostat [9]. For cyclic voltammetry measurements, the NCs were transferred into the ionic liquid.

\subsection{Physical background}

The PL spectra are often used for identification of defects, but, using only this method, it is difficult to address the PL emission to the recombination in a donor or acceptor level. Measurements of charging/recharging processes in an electrochemical cell which contains electrolyte and NCs provide information for suggesting if the trap is a hole one or an electron one, this is a peak of current which appears in a cyclic voltammetry spectrum at positive or negative biases.

In a given neutral NC, the Fermi-level is positioned near the mid-gap, and the energy levels which are below the F-level are filled in with electrons, while those levels which are above the F-level have no electrons, that is, they are empty. When NCs are dispersed in an electrolytic liquid, the common Fermi-level for this system can be considered in respect with the potential of a reference electrode at zero bias $(V=0)$. For example, relative to the $\mathrm{C}$-band of NCs, the position of the F-level can be written as $E_{\mathrm{F}}(0)=E_{c}-1 / 2 E_{g}-\mathrm{e}(\mathrm{V}=0)$. Thus, at positive bias $(+V>0)$, the F-level position will be written as $E_{\mathrm{F}}(+V)=E_{c}-1 / 2 E_{g}-\mathrm{eV}$, and in equilibrium conditions, constant current flows through this system. When positive potential is gradually applied, and an equilibrium condition in the system is not jet reached, the F-level moves toward the C-band of NCs and crosses an empty donor level. At this moment, electrons tunnel from electrolyte to NCs and occupy this energy level, and the measured current reduces. This leads to appearance of a negative peak in the current-voltage spectrum. The greatest peak appears when the F-level crosses the C-band levels, that is, at the potential that corresponds to the energy difference between the initial position of the F-level (without bias) and the C-band edge.

Applied negative potential, vice verse, moves the F-level towards the V-band edge, and makes the acceptor levels empty. This process produces positive peaks in a current-voltage curve, and the largest one corresponds to the V-band edge.

In general, cyclic voltammetry measurements are based on a charging of the NC capacity with electrons or holes, and they can be quantified (see [9-11]).

Electrochemical measurements of the trap location in the bandgap of NCs, offer a unique advantage in determining the traps location relative the $\mathrm{C}(\mathrm{V})$-band edges over photoluminescence measurements, but they are effective slightly above the room temperature. Due to the fact that in NCs the PL spectrum can also be registered in this temperature interval, the data obtained by these two methods are comparable, and they can help 
to determine the donor or acceptor character of the recombination level in $\mathrm{NCs}$ of $\mathrm{CdTe}$ and CdSe.

\section{Results and discussion}

Fig. 1 shows an example of the $80 \mathrm{~K}$ luminescence spectrum of the CdTe NCs samples with $R \approx 3.1 \mathrm{~nm}$ (middle), and $R \approx 1.5 \mathrm{~nm}$ (bottom), as contrasted to that of the bulk CdTe monocrystal (top). The high energy peak in the spectrum of the smallest nanocrystalline sample occurs at $E_{\mathrm{ex}} \approx 2.38 \mathrm{eV}$, its full width at half maximum (FWHM) amounts to $0.21 \mathrm{eV}$. This (inhomogeneous) width is assumed to arise from the radial size distribution $\Delta R / R$ of the CdTe nanospheres. Bigger CdTe NCs with $R \approx 3.1 \mathrm{~nm}$ have a smaller size distribution and a narrower FWHM of about $0.16 \mathrm{eV}$. The bandgap of CdTe at $80 \mathrm{~K}$ is $1.59 \mathrm{eV}$, and the sharp peak at this energy, prominent in the bulk CdTe spectrum, is assigned to excitonic emission. Similar to the bulk PL, exitonic emission is also very prominent in the spectra on CdTe NCs, peaking at $1.8 \mathrm{eV}$ in $\mathrm{CdTe}$ NCs with $R \approx 3.1 \mathrm{~nm}$, and at $2.38 \mathrm{eV}$ in the $R \approx 1.5 \mathrm{~nm}$ CdTe NCs. As compared to bulk CdTe, the excitonic emission of the $R \approx 3.1 \mathrm{~nm}$ CdTe NC sample is shifted to higher energy by $0.30 \mathrm{eV}$, as a result of the quantum confinement, see Fig. 1. Such a large shift for relatively big NCs arises due to the large Bohr exciton radius in CdTe. We underline the appearance in the PL spectra of all three samples of two broad deep level emissions, labelled $E_{1}$ and $E_{2}$, see Fig. 1. Remarkably, they also experience a shift to higher energy. The deep level luminescence bands at $80 \mathrm{~K}$ have been decomposed into two Gaussian bands, $E_{1}$ and $E_{2}$, and numerical results for bulk and nc-CdTe samples are presented in Table 1. These two defect PL bands are already detected in the bulk form of CdTe, but their intensity is substantially enhanced in the unpassivated NCs of CdTe. This is a strong indication that the deep levels responsible arise in CdTe, similar to the results published previously [7], not from impurity, but from the intrinsic lattice defects in $\mathrm{NCs}$, due to much greater surface to volume ratio in NCs.

Fig. 2 demonstrates a typical current-voltage spectrum inferred from the cyclic voltammetry measurements (left panel). On the right panel the energy positions of detected peaks are displayed in regard with the $\mathrm{C}(\mathrm{V})$-band edges of the $R \approx 1.5 \mathrm{~nm} \mathrm{CdTe} \mathrm{NCs}$ (horizontal lines). The quantum energy of radiative transitions from the decomposed PL spectrum, are depicted by the vertical solid lines with an arrow. A good correlation between the quantum energies of optical transitions and the energy difference between the position of voltammetry peaks and the bandgap edges is clear seen. Based on this comparison, we identified two electronic levels located in the bandgap of nc-CdTe, as the donor level, D, and acceptor level, A. The D level is responsible for the near-midgap emission, $E_{2}$, and the $\mathrm{A}$ level is responsible for the emission $E_{1}$. This corresponds to the following case: in the dark the A level is
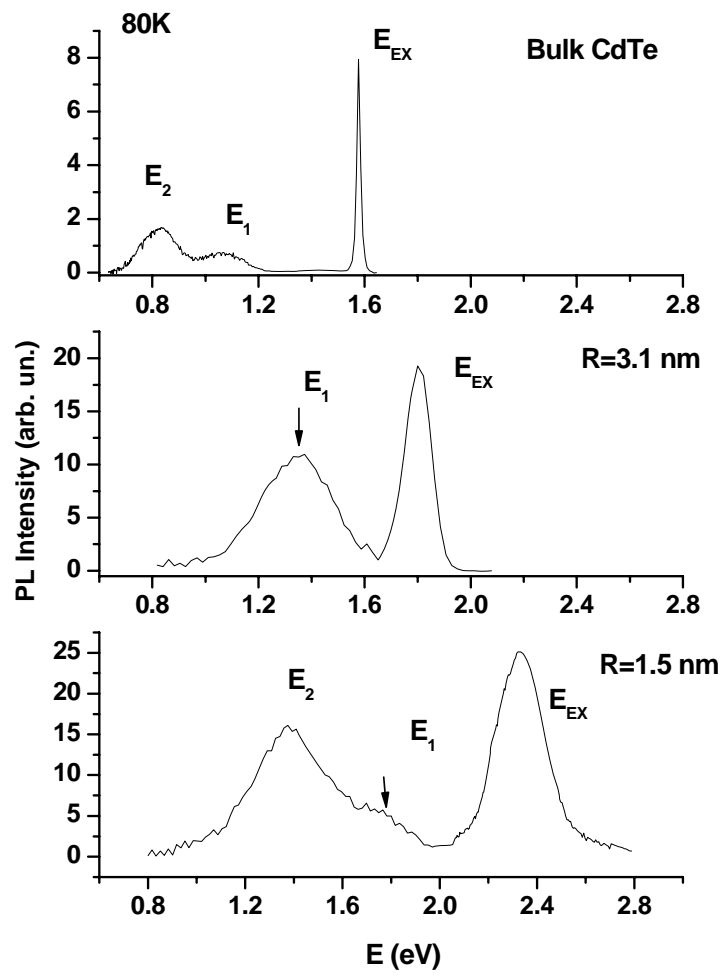

Fig. 1. Luminescence spectrum, at $80 \mathrm{~K}$, of CdTe NCs, $R \approx$ $\approx 1.5 \mathrm{~nm}$ (bottom), $R \approx 3.1 \mathrm{~nm}$ (middle) as contrasted to that of bulk CdTe (top). Note that the intensity of the deep level luminescence $\left(E_{1}, E_{2}\right)$ in the spectrum is expanded by a factor of 5 .

substantially populated with electrons, thus, at illumination, a non equilibrium hole can be captured by this acceptor and recombine with an electron from the Cband. Alternatively, an electron from the C-band can be trapped by the empty D level, and than recombine with the hole trapped in the A level. The rates of radiative recombination through these channels determine the intensities of the $E_{1}$ and $E_{2}$ bands, and when the D level is occupied with electron already in the dark, the $E_{2}$ will be very weak in the spectrum. This case is demonstrated in Fig. 1 (middle) for large CdTe NCs. The reason, why these NCs are charge with electron in the dark, may lead in a specific passivation of the surface depending on the film preparation and illumination; in some cases the $E_{1}$ and $E_{2}$ bands in these NCs have been observed together.

Table 1. Energy and FWHM of the Gaussian peaks in the PL spectra of as-prepared CdSe NCs.

\begin{tabular}{|c|c|c|c|c|c|c|}
\hline & & $E_{\text {ex }}$ & $E_{0}$ & $E_{1}$ & $E_{2}$ & $E_{3}$ \\
\hline \multirow{2}{*}{$\begin{array}{l}\mathrm{CdSe} \\
\mathrm{NCs}\end{array}$} & $\begin{array}{l}\text { PL } \\
\text { spectra }\end{array}$ & 2.07 & 1.85 & 1.57 & 1.3 & 1.05 \\
\hline & FWHM & 0.125 & 0.255 & 0.315 & 0.315 & 0.3 \\
\hline
\end{tabular}




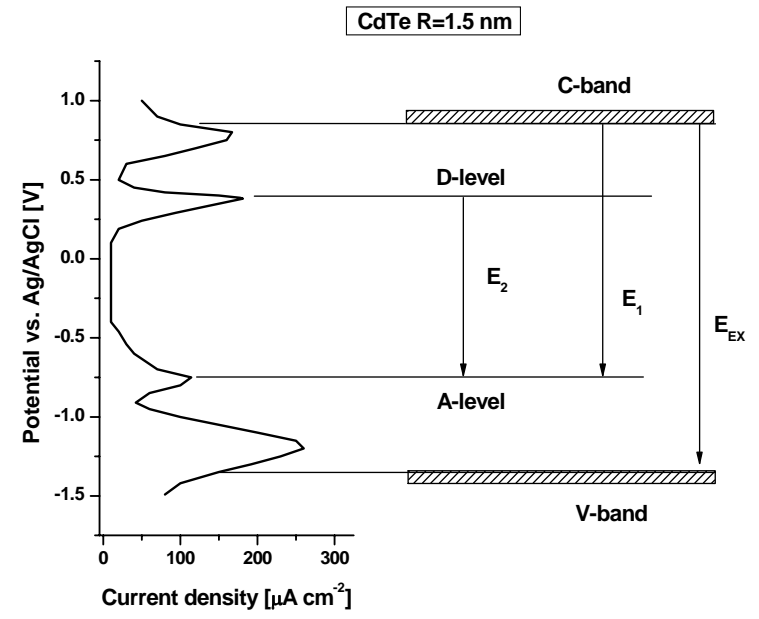

Fig. 2. Current-voltage spectrum inferred from the cyclic voltammetry measurements of CdTe NCs at $300 \mathrm{~K}$ (left panel) and the scheme of energy levels in the bandgap (right panel).

In CdSe NCs, the surface traps emission is known since 90 -th [12]. Since that time, hole traps located at the energy $E_{V}+0.3 \mathrm{eV}$ near the V-band, and deep electron traps at $E_{c}-0.4 \mathrm{eV}$ have been experimentally observed [13]. In this work other than cited above deep traps were studied.

In Fig. 3 presented is the PL spectrum of CdSe NCs with mean $R \approx 2.5 \mathrm{~nm}$. In this spectrum, we found five Gaussian bands with approximately equal full widths at half-maximum (FWHM). The results of the spectral decomposition are summarized in Table 1. Two deeper PL bands, $E_{1}$ and $E_{2}$, are present only in the spectra of the wurtzite type of CdSe NCs, and the corresponding defects have been already identified as two intrinsic D-A pairs: one is located along the hexagonal $c$-axis, the other in the basal Cd-Se bond direction [7]. The deepest band, $\mathrm{E}_{3}$, was clearly observed only at $80 \mathrm{~K}$, and it disappeared with the temperature increase. At RT mainly the $E_{\text {ex }}$ and $\mathrm{E}_{0}$ bands remained in the PL spectrum. This spectrum was analyzed, as in the case of CdTe NCs, together with the data of cyclic voltammetry measurements to determine the donor or acceptor character of the deep levels involved in recombination. In total, two electron and two acceptor traps were determined, which are shown in Table 2.

Table 2. Deep electron and hole traps in CdTe and CdSe NCs.

\begin{tabular}{|l|c|c|c|c|}
\hline & Acceptor & Acceptor & Donor & Donor \\
\hline CdTe NCs & $E_{v}+0.5 \mathrm{eV}$ & & $E_{c}-0.5 \mathrm{eV}$ & \\
\hline CdSe NCs & $E_{v}+$ & $E_{v}+0.8 \mathrm{eV}$ & $E_{c}-$ & $E_{c}-$ \\
& $+0.52 \mathrm{eV}$ & & $-0.25 \mathrm{eV}$ & $-0.65 \mathrm{eV}$ \\
\hline
\end{tabular}

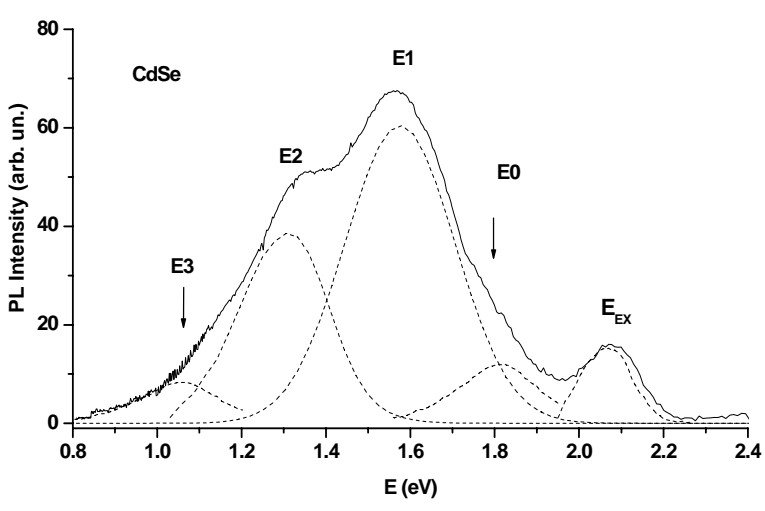

Fig. 3. Low temperature, $80 \mathrm{~K}$, photoluminescent spectra of asprepared CdSe NCs (solid lines). The spectra are decomposed into Gaussian bands (dash lines).

The data obtained in this work support an opinion that surface defects in NCs reduce the QY of excitonic emission. The chemical nature of these defects is still difficult to determine. Taking into account that the acceptor trap at $E_{v}+0.5 \mathrm{eV}$ is commonly present in the CdTe NCs with excess of Te on the surface and in the bulk CdTe grown under the excess of Te, we suggest that this is the $2+/ 1+$ level of $\mathrm{V}_{\mathrm{Cd}}$ or $\mathrm{Te}_{i}$. In addition, the position of this acceptor level in the bandgap of bulk $\mathrm{CdTe}$, which was determined from the PL spectra and electrochemical cyclic voltammetry [9], agrees well with the theoretical predictions of the energy levels of $\mathrm{V}_{\mathrm{Cd}}$ or $\mathrm{Te}_{i}$ [14]. We suggest that a similar defect with an acceptor level at $E_{v}+0.52 \mathrm{eV}$ exists also in CdSe NCs. The deeper acceptor level in CdSe NCs at $E_{v}+0.8 \mathrm{eV}$ may be attributed to the nearest donor-acceptor couple of $\mathrm{V}_{\mathrm{Cd}}$ and $\mathrm{Te}_{i}$ or $\mathrm{V}_{\mathrm{Te}}$.

The donor levels at $E_{c}-0.5 \mathrm{eV}$ in $\mathrm{CdTe}$ and $E_{c}-$ $0.65 \mathrm{eV}$ in CdSe NCs can be tentatively identified according to the [14] as the 2-/1- level of antisite, $\mathrm{Te}_{\mathrm{Cd}}$ in CdTe, and, by analogy, $\mathrm{Se}_{\mathrm{Cd}}$ in CdSe NCs. Such defects exist on the surface of NCs that is enriched with $\mathrm{Te}$ or Se, quite naturally, and the difference between these defects and their bulk analog (surface defects have no one from four bonds with the surrounding $\mathrm{Te}$ or $\mathrm{Se}$ atoms) may not drastically change the electronic energies. This question is still pending for a theoretical solution.

\section{Conclusion}

Defects in CdTe and CdSe NCs have been studied by the photoluminescence combined with electrochemical cyclic voltammetry. Two deep traps, one hole trap and one electron trap, have been detected in CdTe NCs at the energies $E_{v}+0.5 \mathrm{eV}$ and $E_{c}-0.5 \mathrm{eV}$. In CdSe NCs, two 
hole traps have been detected at $E_{v}+0.52 \mathrm{eV}$ and $E_{v}+0.8 \mathrm{eV}$, and two electron traps at $E_{c}-0.25 \mathrm{eV}$ and $E_{c}-0.65 \mathrm{eV}$. We suggest that a native defect, namely, the cation vacancy has an acceptor level at $\approx E_{v}+0.5 \mathrm{eV}$ in CdTe and CdSe NCs. The deeper acceptor level in CdSe NCs at $E_{v}+0.8 \mathrm{eV}$ may be attributed to the nearest couple of an acceptor and donor, $\mathrm{V}_{\mathrm{Cd}}$ and $\mathrm{Te}_{i}$ or $\mathrm{V}_{\mathrm{Te}}$. The deep donor level at $E_{c}-0.5 \mathrm{eV}$ in CdTe and $E_{c}-$ $0.65 \mathrm{eV}$ in CdSe NCs can be tentatively identified as the 2-/1- level of the tellurium (selenium) antisite.

\section{References}

1. Al.L. Efros and A.L. Efros, Interband absorption of light in a semiconductor sphere // Fiz. Tekhn. Poluprovodn. 16(6), p. 1209-1214 (1982) (in Russian); Sov. Phys. - Semiconductor 16(6), p. $772-$ 775 (1982).

2. L.E. Brus, A simple model for the ionization potential, electron affinity, and aqueous redox potentials of small semiconductor crystallites // J. Chem. Phys. 79(11), p. 5566-5571 (1983).

3. F. Wu, J.W. Lewis, D.S. Kliger, and J.Z. Zhang, Unusual excitation intensity dependence of fluorescence of CdTe nanoparticles // J. Chem. Phys. 118 (1), p. 12-16 (2003).

4. U. Woggon, E. Herz, O. Scho1ps, M.V. Artemyev, Ch. Arens, N. Rousseau, D. Schikora, K. Lischka, D. Litvinov and D. Gerthsen, Hybrid epitaxialcolloidal semiconductor nanostructures // Nano Lett. 5(3), p. 485-490 (2005).

5. Atul Konkar, Siyuan Lu, and Anupam Madhukar, Steven M. Hughes and A. Paul Alivisatos, Semiconductor nanocrystal quantum dots on single crystal semiconductor substrates: high resolution transmission electron microscopy // Nano Lett. 5(5), p. 969-973 (2005).

6. P. Swaminathan, V.N. Antonov, J.A.N.T. Soares, J.S. Palmer, and J.H. Weaver, Cd-based II-VI semiconductor nanostructures produced by bufferlayer-assisted growth: structural evolution and photoluminescence // Phys. Rev. B 73(12), p. 125430-125438 (2006).

7. V. Babentsov, J. Riegler, J. Schneider, O. Ehlert, T. Nann, M. Fiederle, Deep level defect luminescence in cadmium selenide nano-crystals films // Journal of Crystal Growth 280 (3-4), p. 502-508 (2005).

8. V. Babentsov, J. Riegler, J. Schneider, M. Fiederle, and T. Nann, Excitation dependence of steady-state photoluminescence in CdSe nanocrystal films // J. Phys. Chem. B 10 (32) p. 15349-15354 (2005).

9. S.K. Poznyak, N.P. Osipovich, A. Shavel, D.V Talapin, M. Gao, A. Eychmuller, and N. Gaponik, Size-dependent electrochemical behavior of thiolcapped CdTe nanocrystals in aqueous solution // J. Phys. Chem. B 109(3), p. 1094-1100 (2005).

10. E. Kuçur, W. Bulcking, R. Giernoth, and Th. Nann, Determination of quantum confinement in $\mathrm{CdSe}$ nanocrystals by cyclic voltammetry // J. Chem. Phys. 119 (4), p. 2333 -2337 (2003).

11. E. Kuçur, W. Bulcking, R. Giernoth, and Th. Nann, Determination of defect states in semiconductor nanocrystals by cyclic voltammetry // J. Phys. Chem. B, 109 (43), p. 20355-20360 (2005).

12. M.G. Bawendi, W.L. Wilson, L. Rothberg, P.J. Carroll, T.M. Jedju, M.L. Steigerwald, L.E. Brus, Electronic structure and photoexited-carrier dynamics in nanometer-size CdSe clusters // Phys. Rev. Lett. 65 (13), p. 1623-1626 (1990).

13. B. Alperson, I. Rubinstein, and G. Hodes, Identification of surface states on individual $\mathrm{CdSe}$ quantum dots by room-temperature conductance spectroscopy // Phys. Rev. B 63 (8), p. 0813031081334 (2001).

14. Su-Huai We and S.B. Zhang, First-principles study of doping limits of CdTe // Phys. status solidi $(b)$ 229 (1), p. 305-310 (2002). 